\title{
D-Serine Is the Dominant Endogenous Coagonist for NMDA Receptor Neurotoxicity in Organotypic Hippocampal Slices
}

\author{
Maria Shleper, Elena Kartvelishvily, and Herman Wolosker \\ Department of Biochemistry, B. Rappaport Faculty of Medicine, Technion-Israel Institute of Technology, Haifa 31096, Israel
}

\begin{abstract}
D-serine occurs at high levels in the brain, where it is an endogenous coagonist at the "glycine site" of NMDA receptors. However, D-serine action has not been previously compared with that of endogenous glycine, and the relative importance of the two coagonists remains unclear. We now investigated the efficiencies of the two coagonists in mediating NMDA receptor neurotoxicity in organotypic hippocampal slices. Removal of endogenous D-serine from slices was achieved by pretreating the tissue with recombinant D-serine deaminase enzyme. This enzyme is several orders of magnitude more efficient than previous methods to remove D-serine. We report that complete removal of D-serine virtually abolished NMDA-elicited neurotoxicity but did not protect against kainate. Although levels of glycine were 10 -fold higher than D-serine, endogenous glycine was ineffective in mediating NMDA receptor neurotoxicity. The effect of endogenous glycine could be observed only after simultaneous removal of endogenous D-serine and blockage of the glycine transporter GlyT1. Our data indicate that D-serine is the dominant coagonist for NMDA receptor-elicited neurotoxicity, mediating all cell death elicited by NMDA in organotypic slices. The results suggest an essential role for this unusual D-amino acid, with implications for the mechanism of neuronal death in the nervous system.
\end{abstract}

Key words: neuromodulation; neurotoxicity; neurotransmission; glutamate; neuroprotection; NMDA receptor

\section{Introduction}

NMDA type of glutamate receptors plays prominent roles in excitatory neurotransmission and is the major culprit in cell death, which occurs after stroke (Danysz and Parsons, 1998). In addition to glutamate, the NMDA receptor requires the obligatory binding of a coagonist to the "glycine site" NMDA receptor 1 (NR1) subunit to mediate ion influx (Johnson and Ascher, 1987). Although glycine was originally suggested as the endogenous coagonist, a number of reports indicate that D-serine is also a relevant endogenous NMDA receptor ligand at the coagonist site (Schell et al., 1995; Mothet et al., 2000).

D-serine is at least as potent as glycine in activating purified recombinant NMDA receptors (Matsui et al., 1995). High levels of D-serine in the brain are generated by activity of the serine racemase enzyme that catalyzes conversion of L- into D-serine (Wolosker et al., 1999; De Miranda et al., 2002). Electrophysiological studies indicate that endogenous D-serine is a coagonist of NMDA receptors by using an enzymatic method to remove D-serine (Mothet et al., 2000). In hippocampal neural cell cultures, pretreatment with D-amino acid oxidase enzyme to deplete endogenous D-serine decreased NMDA receptor responses by $\sim 55 \%$ (Mothet et al., 2000). However, one limitation for the use of D-amino acid oxidase is its inability or low efficiency to remove D-serine in hippocampal slices. Only a minor 19\% decrease in

Received May 10, 2005; accepted Aug. 29, 2005.

This work was supported by the Israel Science Foundation and the Atkins Medical Research Fund for Gerontology.

Correspondence should be addressed to Herman Wolosker at the above address. E-mail: hwolosker@tx.technion.ac.il.

DOI:10.1523/JNEUROSCI.3190-05.2005

Copyright $\odot 2005$ Society for Neuroscience $\quad$ 0270-6474/05/259413-05\$15.00/0 endogenous D-serine was observed after treating hippocampal slices with excess enzyme (Mothet et al., 2000). Additional studies, using D-amino acid oxidase treatment, proposed roles for endogenous D-serine as a coagonist for hippocampal long-term potentiation and NMDA receptor responses in the retina (Stevens et al., 2003; Yang et al., 2003). However, these later studies did not monitor the extent of D-serine depletion by D-amino acid oxidase and incomplete removal of D-serine or artifacts attributable to contaminant proteins in the enzyme preparation cannot be ruled out. Thus, a more efficient method to deplete D-serine in slices is needed to evaluate its physiological role.

In the present study, we sought to compare the roles of endogenous D-serine and glycine in mediating NMDA receptor neurotoxicity in organotypic slice preparations. We developed a new tool to remove D-serine from slices by using the recombinant D-serine deaminase enzyme (DsdA), which is at least three orders of magnitude more efficient than D-amino acid oxidase in destroying D-serine. We show that endogenous glycine plays no significant role in NMDA receptor neurotoxicity in this experimental model. D-serine mediates virtually all NMDA receptorelicited cell death in organotypic hippocampal slices, indicating that D-serine is the dominant and necessary coagonist for NMDA receptor neurotoxicity.

\section{Materials and Methods}

Materials. D-serine, glycine, kainic acid, NMDA, propidium iodide (PI), and $((R)-N$ - 3-(4'-fluorophenyl)-3-(4' phenylphenoxy)) sarcosine hydrochloride (NFPS) were obtained from Sigma (St. Louis, MO). 6,7-Dinitroquinoxaline-2,3-dione (DNQX) and dizocilpine (MK-801) were purchased from Tocris Cookson (Bristol, UK). Basal Medium Eagle, Minimum Essential Medium (MEM), fetal bovine 
serum, and horse serum were obtained from Biological Industries (Kibbutz Beit Haemek, Ashrat, Israel). B27 supplement was obtained from Invitrogen (San Diego, CA). D-amino acid oxidase from pig kidney was obtained from Sigma (lots 104K0937 and 114H9556), Calzyme (lot 4-1-19; Calzyme, San Luis Obispo, CA), and Boehringer Mannheim/ Roche (lot 85236822-64; Boehringer Mannheim, Indianapolis, IN; Roche Products, Welwyn Garden City, UK). Amplex red was obtained from Invitrogen (Eugene, OR).

Organotypic slice cultures. Interface organotypic hippocampal slices were prepared essentially as described previously (Stoppini et al., 1991). Rat pups were killed by quick decapitation following a protocol approved by the Animal Ethics Committee of the Medical School. In brief, hippocampi were harvested from 5- to 7-d-old Sprague Dawley rat pups and sectioned transversely at $400 \mu \mathrm{m}$ using a McIlwain tissue chopper. Three to four slices were then plated onto each $30 \mathrm{~mm}$ culture insert (Millicell-CM; Millipore, Bedford, MA) and maintained in a 5\% $\mathrm{CO}_{2}$ incubator. Culture medium (MEM; supplemented with $25 \mathrm{~mm}$ HEPES, Earle's salts, and 25\% horse serum) was changed twice a week, and the sections were cultured for 12-14 d before treatment.

Recombinant DsdA. DsdA clone from the CFT073 Escherichia coli strain was obtained from Dr. R. Welch (University of Wisconsin-Madison, Madison, WI) and subcloned into pET $28 \mathrm{c}+$ vector (NheI and EcoR1 sites) containing an N-terminal histidine tag. Production of the recombinant protein in E. coli was induced by the addition of $0.8 \mathrm{~mm}$ isopropyl- $\beta$-D-thiogalactopyranoside and affinity purified with nickelnitriloacetic acid agarose beads. After washing in buffer containing 500 $\mathrm{mm} \mathrm{NaCl}$ and $10 \mathrm{~mm}$ imidazol, the protein was eluted with $150 \mathrm{~mm}$ imidazole and dialyzed overnight against PBS. The protein was concentrated to $30-40 \mathrm{mg} / \mathrm{ml}$ with Centriprep (Amicon, Beverly, MA) and frozen at $-70^{\circ} \mathrm{C}$. Deamination of D-serine into pyruvate by DsdA was monitored by a decrease in nicotinamide adenine dinucleotide phosphate $(\mathrm{NADH})$ at $340 \mathrm{~nm}$ as the pyruvate was converted to lactate by added lactate dehydrogenase $(\mathrm{LDH}) . K_{\mathrm{m}}$ and $V_{\max }\left(K_{\mathrm{cat}} \times[\mathrm{E}]\right)$ were calculated by Lineweaver-Burk plots.

$D$-amino acid oxidase activity. Reaction medium contained $20 \mathrm{~mm}$ Tris$\mathrm{HCl}, \mathrm{pH} 7.4,10 \mathrm{U} / \mathrm{ml}$ catalase, $20 \mu \mathrm{g} / \mathrm{ml} \mathrm{D}$-amino acid oxidase (Sigma or Calzyme), $0.2 \mathrm{~mm} \mathrm{NADH}$, and a different concentration of either D-serine or D-alanine. Pyruvate or hydroxypyruvate production was measured by monitoring the decrease in NADH absorbance at $340 \mathrm{~nm}$, as the pyruvate was converted to lactate by LDH.

Recombinant glycine oxidase. Glycine oxidase cDNA from Bacillus subtilis in pET $16 \mathrm{~b}$ vector was a gift from Prof. S. E. Ealick (Cornell University, Ithaca, NY). The recombinant protein was expressed in E. coli and purified as described previously (Settembre et al., 2003).

Determination of D-serine. An aliquot of culture medium $(0.1 \mathrm{ml})$ or cell extract was removed, and the samples were processed for HPLC (De Miranda et al., 2002). It was necessary to routinely treat all samples with DsdA to confirm that the peak that eluted in the position of D-serine corresponded to true D-serine. The choice of the proper culture medium was critical for D-serine determination. MEM-HEPES medium obtained from Invitrogen (lot 3086158) contained a contaminant that totally obscured the real D-serine peak and was not removed by D-serine deaminase treatment. Culture media obtained from Kibbutz Beit Haemek was devoid of contaminant peaks at D-serine elution time and was used in all experiments.

Determination of glycine. Glycine was detected as described previously (Fekkes et al., 1995), with the following modifications: derivatization reagent contained $5 \mathrm{mg}$ of $o$-phthaldialdehyde and $11 \mathrm{~mm}$ $\beta$-mercaptoethanol in $1 \mathrm{ml}$ of methanol. Derivatization was performed in $24 \mathrm{~mm}$ borate buffer, $\mathrm{pH} 9$, for $50 \mathrm{~s}$. Reverse-phase separation of glycine was attained using gradient elution consisting of buffer A $(6.5 \%$ acetonitrile, $3 \%$ tetrahydrofuran, and $0.1 \mathrm{~m} \mathrm{Na}$-acetate, $\mathrm{pH} 6.2$ ) and buffer B (50\% acetonitrile, 3\% tetrahydrofuran, and $0.1 \mathrm{~m}$ Na-acetate, $\mathrm{pH}$ 6.2). Because background peaks in the elution time of glycine were present, confirmation of the identity of the glycine peak was required. This was achieved by treating the samples for $1 \mathrm{~h}$ with glycine oxidase enzyme $(100 \mu \mathrm{g} / \mathrm{ml})$ in $20 \mathrm{~mm}$ borate buffer (final pH 7.5). This enzymatic treatment specifically destroyed all glycine without changing other amino acid levels, allowing accurate determination of true glycine concentration in each sample.

Neurotoxicity experiments. NMDA and kainate-elicited neurotoxicity in hippocampal organotypic slices were performed as described previously (Noraberg et al., 1999), with the following modifications. To elicit excitotoxicity, NMDA $(500 \mu \mathrm{M})$ or kainate $(100 \mu \mathrm{M})$ was added to the medium containing $2 \mu \mathrm{M}$ PI for $3 \mathrm{~h}$. The treatments were performed in at least quadruplicate inserts with three to four slices per insert. Then, the medium was replaced with a new one containing $2 \mu \mathrm{M}$ PI, and cell death was analyzed $24 \mathrm{~h}$ later by visualizing PI fluorescence using an inverted microscope. Pictures at different exposure times were taken, and densitometric analysis was performed by using NIH Image J software as described previously (Noraberg et al., 1999). Maximal PI uptake was determined by adding excess glutamate $(10 \mathrm{~mm})$ to the cultures, which was shown to promote total neuronal death. The values obtained with NMDA and kainate were expressed as a percentage of maximal PI uptake. To remove endogenous D-serine, recombinant DsdA $(10 \mu \mathrm{g} / \mathrm{ml})$ was added to the slice cultures 90 min before the addition of NMDA, which was also present during NMDA insult. The glutamate receptor antagonists MK-801 and DNQX were added 10 min before and during NMDA insult. In some experiments, to evaluate the role of endogenous glycine, slice media was changed after $4 \mathrm{~d}$ in culture for Basal Medium Eagle containing B27 supplement, which lacks serum.

Statistical analysis. Values were analyzed by one-way ANOVA followed by Tukey's multiple-comparison test.

\section{Results}

We sought to evaluate the role of D-serine in NMDA-elicited neurotoxicity in organotypic hippocampal slices. We developed a method to selectively remove $\mathrm{D}$-serine by using recombinant DsdA (EC 4.2.1.14), which has several advantages over D-amino acid oxidase. DsdA is a bacterial enzyme that catalyzes the deamination of D-serine into pyruvate and ammonia. The enzyme is highly specific to D-serine, because L-enantiomers are not substrates under standard conditions (Dupourque et al., 1966). Recombinant DsdA was bacterially expressed and purified to homogeneity (Fig. 1A). We compared the kinetics of DsdA with a commercial preparation of the D-amino acid oxidase, which is known to degrade D-amino acids, generating $\mathrm{H}_{2} \mathrm{O}_{2}$, ammonia, and the corresponding keto-acid (Fig. $1 \mathrm{~B}$ ). The apparent affinity of DsdA for D-serine $\left(K_{\mathrm{m}}=0.1 \mathrm{~mm}\right)$ was more than two orders of 



Figure 2. Endogenous D-serine is essential for NMDA-elicited cell death in organotypic hippocampal slices. $\boldsymbol{A}$, Control (Ctl). $\boldsymbol{B}$, NMDA $(500 \mu \mathrm{m})$ elicited robust cell death in all hippocampal areas as measured by PI uptake. C, Control treated with DsdA (10 $\mu \mathrm{g} / \mathrm{ml}$ for $90 \mathrm{~min}$ ). $\boldsymbol{D}$, Destruction of D-serine by DsdA protected against NMDA-elicited cell death. $\boldsymbol{E}$, NMDA plus MK-801 (30 $\mu \mathrm{m})$ added 10 min before and during the NMDA insult. $\boldsymbol{F}$, NMDA plus DNQX (100 $\mu \mathrm{m})$ added 10 min before and during the NMDA insult. $\mathbf{G}$, Densitometric analysis of $\mathrm{PI}$ uptake and reversal of DsdA effect by addition of $1 \mathrm{~mm}$ glycine (Gly). The values were expressed as a percentage PI uptake relative to that observed with excess L-glutamate (10 mM). The results are average \pm SEM of 4-12 experiments done in quadruplicates. ${ }^{* * *} p<0.001$, different from NMDA insult $(n=12)$.

magnitude higher than that of D-amino acid oxidase under the same experimental conditions $\left(K_{\mathrm{m}}=50 \mathrm{~mm}\right)$. Note that the apparent affinity of $\mathrm{D}$-amino acid oxidase for $\mathrm{D}$-alanine was higher than for D-serine (Fig. $1 B)\left(K_{\mathrm{m}}=10 \mathrm{~mm}\right)$. The low affinity of $\mathrm{D}$-amino acid oxidase for $\mathrm{D}$-serine at neutral $\mathrm{pH}$ is in agreement with a previous study (D'Aniello et al., 1993). The catalytic efficiencies calculated by the $K_{\text {cat }} / K_{\mathrm{m}}$ ratio were $157 \mathrm{mM}^{-1} \cdot \mathrm{s}^{-1}$ and $0.025 \mathrm{~mm}^{-1} \cdot \mathrm{s}^{-1}$ for DsdA and D-amino acid oxidase, respectively. Thus, the overall efficiency of recombinant DsdA was more than three orders of magnitude higher than D-amino acid oxidase. Contrasting with the purity of DsdA (Fig. 1A), analysis of commercial D-amino acid oxidase preparations revealed varying degrees of impurities (supplemental Fig. 1, available at www.jneurosci.org as supplemental material). Only the D-amino acid oxidase from Boehringer Mannheim/Roche was relatively pure, but this preparation is no longer commercialized. In addition, batches of D-amino acid oxidase contain significant amounts of D-aspartate oxidase enzyme activity that quickly destroys NMDA (supplemental Fig. 1, available at www.jneurosci.org as supplemental material).

Organotypic hippocampal slices maintained for $12 \mathrm{~d}$ in culture contained D-serine at a concentration of $0.42 \pm 0.07$ $\mathrm{nmol} / \mathrm{mg}$ slice protein (average $\pm \mathrm{SEM} ; n=4$ ). D-serine was also present in conditioned media of the slices at $1.6 \pm 0.6 \mu \mathrm{M}(n=7)$. Addition of DsdA efficiently depleted D-serine from the slices (Fig. $1 C$ ) and culture medium (Fig. 1D). Levels of L-serine and other amino acids were unchanged (data not shown), indicating that DsdA can be used as a tool to study the actions of endogenous D-serine in this preparation.

To verify the role of endogenous D-serine in neurotoxicity, hippocampal slices were first exposed to $0.5 \mathrm{~mm} \mathrm{NMDA}$, and cell death was monitored by analyzing PI uptake, which labels nuclei of dead cells (Tasker et al., 1992). Control slices exhibited minimal or no PI fluorescence (Fig. 2A), whereas addition of NMDA elicited robust PI uptake in all hippocampal regions (Fig. $2 B$ ).
Pretreatment of the cultures with DsdA to remove endogenous D-serine completely prevented cell death elicited by NMDA (Fig. 2D). In the absence of NMDA, DsdA had no effect (Fig. 2C). Addition of MK801, a noncompetitive antagonist of NMDA type of glutamate receptors, also prevented cell death, confirming the role of NMDA receptors in excitotoxicity (Fig. $2 E)$. DNQX, an antagonist of AMPA/kainate type of glutamate receptors, had no effect (Fig. $2 F$ ).

Densitometric analysis of PI uptake revealed that DsdA treatment promoted complete neuroprotection against NMDA, which was identical to that promoted by MK-801 (Fig. 2G). If the DsdA action is specific, stimulation of NMDA receptors by another coagonist should reverse its effects. Accordingly, the effect of DsdA was counteracted by adding exogenous $1 \mathrm{~mm}$ glycine, which maximally stimulates NMDA receptors at this very high and nonphysiological concentration (Fig. $2 G)$. The reversibility of the DsdA effect indicates that its action is not a result of a nonspecific inhibition of NMDA receptor activity. NMDA receptors were already saturated with endogenous D-serine under our experimental conditions, because no additional neurotoxicity was observed by adding exogenous glycine or D-serine (Fig. 2G) (data not shown).

To confirm the specificity of DsdA action, slice cultures were incubated with kainate that elicits neurotoxicity by an NMDA receptor-independent mechanism. The kainate effect was blocked by the antagonist DNQX but was unaffected by DsdA or MK-801 (Fig. 3A-D). Densitometric analysis of PI uptake confirmed the lack of effect of DsdA in kainate-evoked neurotoxicity (Fig. 3E).

The prevailing action of endogenous D-serine in NMDAelicited neurotoxicity is not a result of the absence of glycine from the cultures. To determine the endogenous levels of glycine, we used a serum-free culture medium that lacks exogenous glycine. Glycine concentration measured in conditioned media at culture day 12 was $19.8 \pm 1.8 \mu \mathrm{M}(n=4), \sim 10$-fold higher than endogenous $\mathrm{D}$-serine at the same culture age. Despite the high-glycine values present in the medium, removal of endogenous D-serine by DsdA completely abolished neurotoxicity, indicating that endogenous glycine is functionally much less effective than D-serine (Fig. 4). To evaluate the potency of glycine, we first treated organotypic slices with DsdA to remove all D-serine and then exposed the slices to NMDA plus increasing amounts of exogenous glycine (Fig. 4A-D). A significant increase in NMDA-elicited toxicity was only observed at total glycine concentrations several-fold higher than endogenous glycine ( $80-150 \mu \mathrm{M}$ total glycine) (Fig. $4 E)$.

One possibility to explain the ineffectiveness of endogenous glycine in mediating NMDA-elicited neurotoxicity is the action of the glycine transporter (GlyT1), previously shown to effectively limit the synaptic glycine concentration (Berger et al., 1998; Chen et al., 2003). Thus, we checked whether the glycine transporter was involved in preventing the endogenous glycine effect by adding NFPS, a specific inhibitor of the GlyT1 transporter. NMDA-elicited neurotoxicity was not potentiated by NFPS when 
endogenous D-serine was present (Fig. $4 F$ ). After D-serine was removed by DsdA treatment, NFPS elicited a potentiation of NMDA-elicited cell death (Fig. $4 F$ ). Thus, endogenous glycine was able to mediate NMDA receptor neurotoxicity only after GlyT1 was blocked and all endogenous D-serine had been destroyed.

NR3A/B subunits of NMDA receptors form a relatively $\mathrm{Ca}^{2+}$-impermeable channel that is activated by glycine and inhibited by D-serine when coexpressed with NR1 (Chatterton et al., 2002). The NR3 subunit is expressed in the slices, its levels being lower than those after initial plating (supplemental Fig. 2, available at www. jneurosci.org as supplemental material). This may reflect the dramatic decrease in hippocampal NR3A mRNA (Ciabarra et al., 1995) and protein (Sasaki et al., 2002) that occurs during or after the second postnatal week. Because NR3 expression is still detectable in slices after $10 \mathrm{~d}$ in culture, this preparation may be useful to study the role of NR3 subunits. It is noteworthy that the receptors formed by the NR1 and NR3 subunits are insensitive to glutamate, NMDA, or MK-801 (Chatterton et al., 2002). This suggests that NR3 receptors do not mediate the NMDAelicited neurotoxicity seen in the present study.

\section{Discussion}

In the present study, we demonstrated that $\mathrm{D}$-serine is the major and necessary endogenous NMDA receptor coagonist in a neurotoxicity model, mediating virtually all NMDA receptor-elicited neuronal death. It has been shown that endogenous D-serine mediates approximately half of NMDA receptor responses in neural cultures, whereas only a $25 \%$ decrease in NMDA receptor responses was detected in hippocampal slices (Mothet et al., 2000). The modest effect of $\mathrm{D}$-amino acid oxidase to remove D-serine from slices has been attributed to limited penetration of the enzyme in the tissue (Mothet et al., 2000). A partial protection against NMDA toxicity by D-amino acid oxidase treatment in corticostriatal slices was reported recently (Katsuki et al., 2004). In this preliminary account, the authors did not monitor endogenous D-serine or glycine levels and provided no indication that the treatment decreased D-serine. The use of batches of D-amino acid oxidase of unknown purity complicates the analysis of NMDA-elicited neurotoxicity, because they display contaminant D-aspartate oxidase activity that destroys NMDA (supplemental Fig. 1, available at www.jneurosci.org as supplemental material). In the present study, we overcame these caveats by establishing a highly effective method to remove D-serine with the use of DsdA. The depletion of D-serine from the slices is probably attributable to efficient destruction of released D-serine, resulting in depletion of intracellular stores that are not readily replenished by the biosynthetic

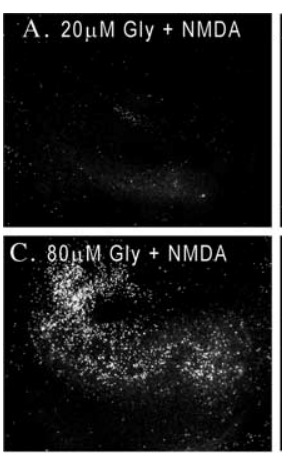

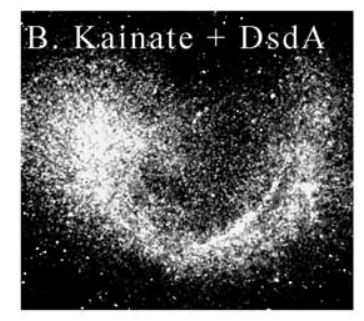

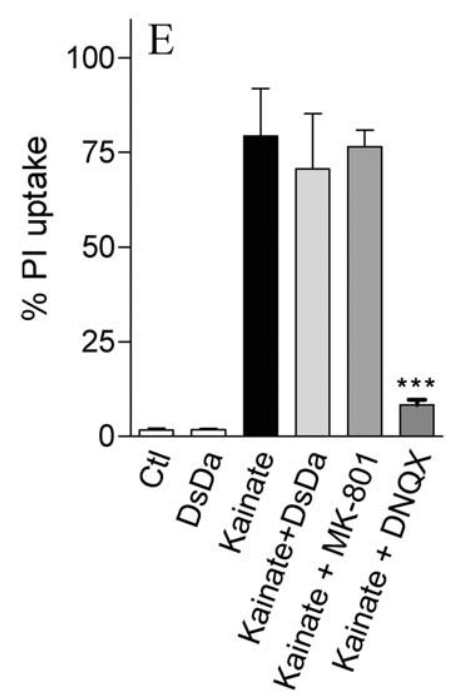

Endogenous D-serine is not required for kainate-elicited cell death. Conditions were as in Figure 2, except that 列 $100 \mu$ DNQX. D, Kainate plus $30 \mu$ MK-801. E, Densitometric analysis of kainate-elicited neurotoxicity. Ctl, Control. The results are average \pm SEM of six experiments done in triplicates from a different slice preparation. ${ }^{* * *} p<0.001$, different
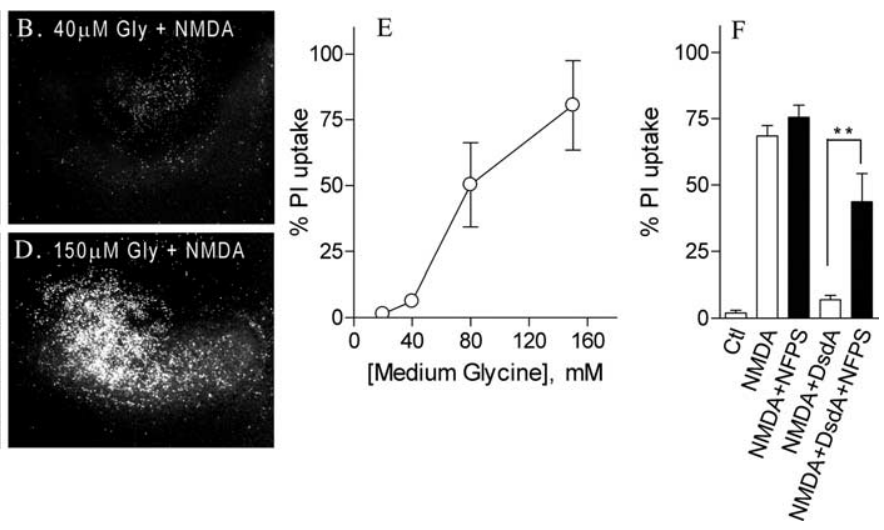

Figure 4. Glycine dependence for NMDA neurotoxicity in the absence of D-serine and potentiation by GlyT1 inhibitor. A-D, Effect of glycine (Gly) in NMDA-elicited neurotoxicity in the absence of endogenous D-serine. Slices were pretreated with DsdA (10 $\mu \mathrm{g} / \mathrm{ml}$ for $90 \mathrm{~min}$ ) and exposed to $500 \mu \mathrm{m}$ NMDA as described in Figure 2. Basal glycine $(\boldsymbol{A})$ accounted for $\sim 20 \mu \mathrm{m}$, as measured exposed to NMDA. $\boldsymbol{F}$, Effect of GlyT1 inhibitor on NMDA-elicited neurotoxicity in the absence or presence of DsdA. NFPS (1 $\mu \mathrm{m})$ was present 30 min before and during NMDA insult. Ctl, Control. ${ }^{* *} p<0.01$, significant difference.

enzyme. The lower thickness of organotypic slices (150 $\mu \mathrm{m}$ thick) after 2 weeks in culture (Stoppini et al., 1991) may also facilitate complete removal of $\mathrm{D}$-serine.

The amount of endogenous glycine in conditioned culture media was at least 10 times higher than D-serine. Nevertheless, endogenous glycine was functionally inefficient to mediate neurotoxicity, unless the glycine transporter GlyT1 was blocked and D-serine was removed. Because the affinity of purified NMDA receptors to glycine is similar to D-serine (Matsui et al., 1995), failure of endogenous glycine to mediate NMDA neurotoxicity is probably attributable to the efficient glycine uptake that limits glycine access to NMDA receptor sites (Berger et al., 1998). This is supported by the observation that exogenous glycine activated NMDA receptors only when supplemented at very high and nonphysiological values, probably required to overcome the glycine transporter. 
In our experimental model, no additional neurotoxicity was observed by adding exogenous D-serine, suggesting that endogenous D-serine was saturating. However, because we do not monitor NMDA receptor neurotransmission, it is not possible to infer the true saturation of these receptors by D-serine. The effect of exogenous D-serine during normal NMDA neurotransmission may be different from that observed in neurotoxicity.

Recently, vesicular release of D-serine elicited by AMPA receptor activation has been demonstrated in glial cultures (Mothet et al., 2005). This provides a strong indication that D-serine is a glia-derived transmitter. However, it is not clear whether vesicular release of D-serine takes place during NMDA receptor-elicited neurotoxicity. We did not observe any protective effect of AMPA receptor blockage in NMDA receptor neurotoxicity (Fig. 2). It is possible that, under our experimental conditions, a significant fraction of released D-serine is mediated by reversal of the neutral amino acid transporter previously demonstrated in glial cultures (Ribeiro et al., 2002).

In summary, the present study points to a previously undescribed dominant role for D-serine in NMDA receptor neurotoxicity. The affinity of neutral amino acid transporters for D-serine is low, contrasting the high affinity of GlyT1 for glycine (Ribeiro et al., 2002). Thus, it is conceivable that D-serine more easily diffuses to extrasynaptic locations and activates NMDA receptors that lie far from the release sites. This will provide a more general activation of NMDA receptors, which may play a crucial role in the massive activation that occurs in neurotoxicity. Our data have implications for stroke treatment, in which blockers of D-serine synthesis by serine racemase may be useful as neuroprotective agents.

\section{References}

Berger AJ, Dieudonne S, Ascher P (1998) Glycine uptake governs glycine site occupancy at NMDA receptors of excitatory synapses. J Neurophysiol 80:3336-3340.

Chatterton JE, Awobuluyi M, Premkumar LS, Takahashi H, Talantova M, Shin Y, Cui J, Tu S, Sevarino KA, Nakanishi N, Tong G, Lipton SA, Zhang D (2002) Excitatory glycine receptors containing the NR3 family of NMDA receptor subunits. Nature 415:793-798.

Chen L, Muhlhauser M, Yang CR (2003) Glycine tranporter-1 blockade potentiates NMDA-mediated responses in rat prefrontal cortical neurons in vitro and in vivo. J Neurophysiol 89:691-703.

Ciabarra AM, Sullivan JM, Gahn LG, Pecht G, Heinemann S, Sevarino KA (1995) Cloning and characterization of chi-1: a developmentally regulated member of a novel class of the ionotropic glutamate receptor family. J Neurosci 15:6498-6508.

D'Aniello A, Vetere A, Petrucelli L (1993) Further study on the specificity of D-amino acid oxidase and D-aspartate oxidase and time course for complete oxidation of D-amino acids. Comp Biochem Physiol B 105:731-734.

Danysz W, Parsons AC (1998) Glycine and N-methyl-D-aspartate receptors: physiological significance and possible therapeutic applications. Pharmacol Rev 50:597-664.

De Miranda J, Panizzutti R, Foltyn VN, Wolosker H (2002) Cofactors of serine racemase that physiologically stimulate the synthesis of the
$N$-methyl-D-aspartate (NMDA) receptor coagonist D-serine. Proc Natl Acad Sci USA 99:14542-14547.

Dupourque D, Newton WA, Snell EE (1966) Purification and properties of D-serine dehydrase from Escherichia coli. J Biol Chem 241:1233-1238.

Fekkes D, van Dalen A, Edelman M, Voskuilen A (1995) Validation of the determination of amino acids in plasma by high-performance liquid chromatography using automated pre-column derivatization with o-phthaldialdehyde. J Chromatogr B Biomed Appl 669:177-186.

Johnson JW, Ascher P (1987) Glycine potentiates the NMDA response in cultured mouse brain neurons. Nature 325:529-531.

Katsuki H, Nonaka M, Shirakawa H, Kume T, Akaike A (2004) Endogenous D-serine is involved in induction of neuronal death by $\mathrm{N}$-methyl-Daspartate and simulated ischemia in rat cerebrocortical slices. J Pharmacol Exp Ther 311:836-844.

Matsui T, Sekiguchi M, Hashimoto A, Tomita U, Nishikawa T, Wada K (1995) Functional comparison of D-serine and glycine in rodents: the effect on cloned NMDA receptors and the extracellular concentration. J Neurochem 65:454-458.

Mothet JP, Parent AT, Wolosker H, Brady Jr RO, Linden DJ, Ferris CD, Rogawski MA, Snyder SH (2000) D-serine is an endogenous ligand for the glycine site of the N-methyl-D-aspartate receptor. Proc Natl Acad Sci USA 97:4926-4931.

Mothet JP, Pollegioni L, Ouanounou G, Martineau M, Fossier P, Baux G (2005) Glutamate receptor activation triggers a calcium-dependent and SNARE protein-dependent release of the gliotransmitter D-serine. Proc Natl Acad Sci USA 102:5606-5611.

Noraberg J, Kristensen BW, Zimmer J (1999) Markers for neuronal degeneration in organotypic slice cultures. Brain Res Brain Res Protoc 3:278-290.

Ribeiro CS, Reis M, Panizzutti R, de Miranda J, Wolosker H (2002) Glial transport of the neuromodulator D-serine. Brain Res 929:202-209.

Sasaki YF, Rothe T, Premkumar LS, Das S, Cui J, Talantova MV, Wong HK, Gong X, Chan SF, Zhang D, Nakanishi N, Sucher NJ, Lipton SA (2002) Characterization and comparison of the NR3A subunit of the NMDA receptor in recombinant systems and primary cortical neurons. J Neurophysiol 87:2052-2063.

Schell MJ, Molliver ME, Snyder SH (1995) D-serine, an endogenous synaptic modulator: localization to astrocytes and glutamate-stimulated release. Proc Natl Acad Sci USA 92:3948-3952.

Settembre EC, Dorrestein PC, Park JH, Augustine AM, Begley TP, Ealick SE (2003) Structural and mechanistic studies on ThiO, a glycine oxidase essential for thiamin biosynthesis in Bacillus subtilis. Biochemistry 42:2971-2981.

Stevens ER, Esguerra M, Kim PM, Newman EA, Snyder SH, Zahs KR, Miller RF (2003) D-serine and serine racemase are present in the vertebrate retina and contribute to the physiological activation of NMDA receptors. Proc Natl Acad Sci USA 100:6789-6794.

Stoppini L, Buchs PA, Muller D (1991) A simple method for organotypic cultures of nervous tissue. J Neurosci Methods 37:173-182.

Tasker RC, Coyle JT, Vornov JJ (1992) The regional vulnerability to hypoglycemia-induced neurotoxicity in organotypic hippocampal culture: protection by early tetrodotoxin or delayed MK-801. J Neurosci 12:4298-4308.

Wolosker H, Blackshaw S, Snyder SH (1999) Serine racemase: a glial enzyme synthesizing D-serine to regulate glutamate- $N$-methyl-D-aspartate neurotransmission. Proc Natl Acad Sci USA 96:13409-13414.

Yang Y, Ge W, Chen Y, Zhang Z, Shen W, Wu C, Poo M, Duan S (2003) Contribution of astrocytes to hippocampal long-term potentiation through release of D-serine. Proc Natl Acad Sci USA 100:15194-15199. 